\title{
CAMBIOS EN LA RESISTENCIA COMPRESIVA DEL IONÓMERO DE VIDRIO AL SER GRABADO CON ÁCIDO ORTOFOSFÓRICO
}

\author{
Stefany Gil García, est. odontología ${ }_{1}$, Sara Mosquera Arenas, est. odontología 1, \\ Luz Adriana Hoyos Arias, est. odontología , Tatiana Domínguez Jiménez, est. odontología ${ }_{1}$, \\ Luz Mónica Arango B., Esp. ${ }_{1}$, Clara Liliana Gallego G. , Esp. ${ }_{1}$
}

${ }_{1}$ Facultad de Odontología, Universidad Cooperativa de Colombia, sede Medellín, Colombia

Recibido: 20 de noviembre del 2012. Aprobado: 14 de febrero del 2013.

*Autor de correspondencia: Clara Gallego G., Facultad de Odontología, Universidad Cooperativa de Colombia, Medellín, Colombia, (57) 42706466 , Carrera 47 n. ${ }^{\circ} 37$ Sur-18, correo electrónico: clara.gallego@campusucc.edu.co

Cómo citar este artículo: Gil García S, Mosquera Arenas S, Hoyos Arias LA, Domínguez Jiménez T, Arango B. LM, Gallego G. CL. Cambios en la resistencia compresiva del ionómero de vidrio al ser grabado con ácido ortofosfórico. Revista Nacional de Odontología. 2013; 9(16): 67-73.

Resumen. Introducción: los ionómeros de vidrio resinomodificados son materiales usados como base intermedia en procedimientos dentales. El objetivo fue evaluar los cambios que ocurren en la resistencia compresiva de un ionómero de vidrio resinomodificado después de ser grabado con ácido ortofosfórico al 37\%. Materiales y métodos: Estudio experimental in vitro. Se prepararon 40 moldes termoformados, 20 muestras en el grupo experimental (GE) a las que se les aplicó ácido fosfórico durante $15 \mathrm{~s}$ y fueron lavadas durante 40 s, y 20 muestras en el grupo control (CG). Diez muestras de cada grupo se almacenaron por 48 horas al baño maría. Todas las muestras fueron sometidas a una fuerza compresiva usando una máquina universal de prueba. El análisis se realizó en el programa estadístico sPss versión 19. Se realizaron las pruebas de Tukey y Anova. Resultados: hubo diferencias estadísticamente significativas entre los grupos con y sin ácido a los 15 minutos con respecto a los grupos con y sin ácido a las 48 horas $(\mathrm{P}=0,01)$. Conclusiones: la aplicación de ácido fosfórico al $37 \%$ no afecta la resistencia compresiva de los ionómeros de vidrio resinomodificados. La reducción en la resistencia compresiva podría estar asociada al almacenamiento en medio húmedo.

Palabras clave: ácido ortofosfórico, base intermedia, fuerza compresiva, ionómero de vidrio resino modificado.

Changes in the Resistance to Compression of Resin-modified Glass after Etching with Orthophosphoric Acid

Abstract. Introduction: Resin-modified glass ionomers are materials used as an intermediate base in some dental procedures. Our objective was to evaluate the changes that occur in resistance to compression in a light-cured resin-modified glass ionomer after etching it with $37 \%$ orthophosphoric acid. Materials and methods: We carried out an experimental study in vitro. We prepared forty thermoformed molds. The control group (CG) consisted of 20 samples to which no phosphoric acid was applied and the experimental group (GE) included 20 samples that were etched with $37 \%$ orthophosphoric acid for 15 seconds and rinsed with water for 40 seconds. All samples were subjected to a compressive test using an Instron universal testing machine. 10 samples of each group were stored in a water bath. For the analysis we used spss version 19. Tukey and Anova tests were performed. Results: There were statistically significant differences between the groups with and without acid at 15 minutes with respect to the groups with and without acid at 48 hours. $(\mathrm{P}=0.01)$. Conclusions: The application of $37 \%$ phosphoric acid for 15 seconds does not affect the resistance to compression strength of a lightcured resin-modified glass ionomer. Reduction in resistance to compression might be associated with storing the samples in a wet environment.

Keywords: orthophosphoric acid, intermediate base, resistance to compression, light-cured resin-modified glass ionomer.

\section{Mudanças na resistência compreensiva do ionômero de vidro ao ser gravado com ácido ortofosfórico}

Resumo. Introdução: os ionômeros de vidro resinomodificados são materiais usados como base intermediária em procedimentos dentais. $\mathrm{O}$ objetivo foi avaliar as mudanças que ocorrem na resistência compreensiva de um ionômero de vidro resinomodificado depois de ser gravado com ácido ortofosfórico a $37 \%$. Materiais e métodos: estudo experimental in vitro. Prepararam-se 40 moldes termoformados, 20 amostras no grupo experimental (GE), às quais foi aplicado ácido fosfórico durante $15 \mathrm{~s}$ e foram lavadas durante 40 s, e 20 amostras no grupo controle (GC). Dez amostras de cada grupo se armazenaram por 48 horas em banho-maria. Todas as amostras foram submetidas a uma forca compreensiva ao usar uma máquina universal de prova. A análise se realizou no programa estatístico spss versão 19. Realizaram-se as provas de Tukey e Anova. Resultados: houve diferenças estatisticamente significativas entre os grupos com e sem ácido aos 15 minutos com relação aos grupos com e sem ácido às 48 horas $(\mathrm{P}=0,01)$. Conclusões: a aplicação de ácido fosfórico a $37 \%$ não afeta a resistência compreensiva dos ionômeros de vidro resinomodificados. A redução na resistência compreensiva poderia estar associada ao armazenamento em meio úmido.

Palavras-chave: ácido ortofosfórico, base intermediária, força compreesiva, ionômero de vidro resinomodificado. 


\section{Introducción}

En los últimos años, las propiedades físico-químicas de los materiales que se utilizan en odontología han hecho que la práctica clínica sea más segura tanto para los odontólogos como para los pacientes. El ionómero de vidrio es uno de los materiales más utilizados en el área de rehabilitación oral, y actualmente es el material de elección como base intermedia debido a su adhesión a la estructura dentaria y a su efecto anticariogénico, además de su comportamiento mecánico [1-5]. Su elevado módulo elástico y su coeficiente de expansión térmica son similares a los de la dentina, lo que le da una rigidez similar a ella [6-8]. Por la liberación de fluoruros, se consideran como uno de los materiales más completos del arsenal restaurador; también catalogados como "materiales inteligentes" por su capacidad de prevenir la caries secundaria relacionada a una baja en el pH [9]. Estas condiciones serán propicias si se realiza una correcta manipulación del material y un pulido adecuado, debido a que se incrementará la liberación del fluoruro durante un periodo de 12-18 semanas, localizándose en la estructura dentaria. Tanto el esmalte como el cemento pueden absorber cantidades sustanciales de flúor, gracias al íntimo contacto molecular que facilita el intercambio de fluoruros $[10,11]$.

Entre las propiedades del ionómero de vidrio se encuentran:

1. Biológicas: el intercambio iónico con la estructura dentaria, que se obtiene a partir del ácido polialquenoico y la liberación de fluoruro para mejorar la remineralización [12].

2. Químicas: su capacidad de adhesividad al cemento, la dentina y el esmalte, así como a elementos metálicos. No provoca reacciones pulpares significativas y posee baja acidez y solubilidad a los fluidos bucales.

3. Mecánicas: resistencia a la compresión y a la tensión, además de su resistencia al desgaste y a la erosión. Al ser grabados se producen en su superficie micro-retenciones similares a las que se producen en el esmalte.

Lo anterior hace que el ionómero de vidrio sea el material de elección para ser utilizado como protector dentino-pulpar en cavidades profundas en operatoria dental, ya que además de evitar la micro-filtración de bacterias hacia el complejo dentino-pulpar, actúa como sustituto de dentina evitando la flexión del techo came- ral durante la condensación del material restaurador definitivo [8]. En su proceso de maduración, las etapas por las que pasa el material son: la inicial, que se produce dentro de los primeros 10 minutos después de la mezcla, y la final, que supone la liberación del calcio y cationes de aluminio dentro de la matriz; es un proceso lento y continuo a largo plazo de la reacción ácido-base [13].

Durante la primera reacción, el material es muy sensible a la absorción de agua, mientras que durante el segundo paso el material es muy susceptible a la deshidratación [13]. Los ionómeros de vidrio presentan una alta susceptibilidad a la humedad debido a su proceso de endurecimiento, el cual tiene una maduración inicial durante las primeras 24 horas [1-14].

La preparación del cemento de ionómero de vidrio convencional consiste en la mezcla de dos componentes, polvo y líquido. El polvo es un vidrio especial, compuesto básicamente de flúor, aluminio y silicio, que debe sus propiedades opalescentes a la presencia de fluoruro de calcio. El líquido, que es una solución electrolítica de copolímeros con radicales carboxilo, recibe el nombre químico de ácido polialquenoico [15]. La sensibilidad a la humedad del ionómero de vidrio comienza durante la primera fase reactiva y durante el secado, ya que el material se endurece después del ajuste inicial, motivo por el cual estos pueden perder muchas de sus propiedades en razón a que la reacción de endurecimiento y secado del ionómero ocurre en cuatro etapas, que son:

- Formación de la sal.

- Transformación de sol/gel.

- Endurecimiento (continuación del sol/formación de gel).

- Hidratación con $\mathrm{H}_{2} \mathrm{O}$ obligando a una sal, los grupos de ácido y a un gel [16].

Por este motivo se han realizado modificaciones en la composición de los ionómeros convencionales, agregándoles partículas de monómeros hidrofílicos que disminuyen la susceptibilidad inicial del cemento a la humedad [17]. Los ionómeros de vidrio resino modificados constituyen uno de los últimos materiales incorporados como bases intermedias, los cuales fueron introducidos en el mercado entre los años 1993 y 1994, pudiendo ser utilizados como materiales de restauración definitiva. Su composición, compatibilidad biológica y acción adhesiva a la dentina y al esmalte son muy similares a los ionómeros convencionales [18]. Sus mejores propiedades mecánicas le permiten ser grabado 
[16-19]. La sensibilidad a corto plazo por el agua resulta del ablandamiento de la superficie y, como consecuencia, una baja resistencia al desgaste, lo que limita el potencial del cemento ionómero de vidrio para las aplicaciones dentales [13].

Según la adhesión de los ionómeros de vidrio, este posee iones lixiviables, aquellos que son arrastrados por el agua, y el ácido polialquenoico, que permite adherencia a la estructura dental, liberación de flúor y una buena biocompatibilidad; poseen baja resistencia a la tracción y a la fractura, son susceptibles al ataque por la humedad durante el periodo de ajuste inicial y tienen un corto tiempo de trabajo [20].

También es importante destacar que tanto la resistencia a la compresión y a la tensión, como la resistencia al desgaste y a la erosión tienen unos valores aceptables [21], teniendo en cuenta que la durabilidad del material está influida por la inapropiada preparación del cemento, la inadecuada protección de la restauración y las constantes variaciones del medio oral, como son la humedad y la temperatura [10].

El grado de desmineralización depende de varios parámetros; por ejemplo: el tipo de ácido (en este caso el ácido ortofosfórico), las características específicas de cada ácido (concentración, pH y aglutinante), el tiempo de grabado (desde 15 hasta 120 segundos), la presentación del ácido (gel, semi-gel o solución acuosa) y el tiempo de lavado [22].

En el artículo: "Técnica Sandwich con acondicionamiento ácido selectivo empleando ionómeros", se creó cierta controversia sobre el uso del ácido en el ionómero de vidrio y cómo podría este afectarlo, observando que al realizar el acondicionamiento ácido sobre el ionómero de vidrio, utilizando el protocolo de adhesión de las resinas compuestas, lo que implica aplicación de ácido ortofosfórico, lavado y secado, se pone en riesgo la integridad del ionómero convencional de base, sobre todo si se lleva a cabo en el momento en el que el ionómero está en el proceso de fraguado y es químicamente más reactivo y susceptible a la disolución [9].

Mallman y colaboradores (2007) [21] reportaron que los ionómeros de vidrio resino-modificados mostraron valores muy altos en la resistencia compresiva con respecto a los ionómeros convencionales, muy probablemente por sus componentes resinosos.

Della y colaboradores (2007) [23] concluyeron que los ionómeros de vidrio resino-modificados fueron más efectivos en la prevención de la penetración de colorante en la interfase resina-ionómero resino-modificado y dentina, que en el ionómero convencional.
Según un artículo publicado por Anstice y colaboradores (1992) [24], los criterios de resistencia compresiva de los ionómeros de vidrio especificados en la norma Iso de 1986 y en los estándares británicos BSI, 1981, establecen que la resistencia compresiva mínima aceptable de estos materiales debe ser no menor a 65 megapascales (Mpa) para los ionómeros tipo 1 o cementantes, y no menor a $125 \mathrm{Mpa}$ para los ionómeros tipo 2 o restaurativos [24].

Durante la reacción ácido base de los ionómeros de vidrio es importante el papel que desempeña el agua, puesto que por medio de esta se da la reacción, además de que queda integrada dentro de la matriz para crear una estructura estable $[1,14,25]$.

Igualmente, se observó que la resistencia de los ionómeros de vidrio resino-modificados aumentó, cuando Mitra, en 1991 [22], describe la reacción de los cementos de curado por luz, en cuatro etapas: tres de ellas incluyen la reacción ácido-básica tradicional, y el cuarto paso la reacción de curado por la luz, que involucra la polimerización de los grupos metacrilato que origina el que se desarrolle un material firmemente estructurado de aspecto coriáceo [22].

Se ha demostrado que un nuevo sistema de ionómero de vidrio formado por una resina de fotocurado con partículas de nanorrelleno ha mejorado la resistencia al desgaste y la dureza de las restauraciones, las que anteriormente mostraban a largo plazo una menor resistencia a fuerzas compresivas, rápida abrasión de la superficie, filtración marginal y regular apariencia estética [26].

En los ionómeros convencionales, la superficie del cemento se manifiesta clínicamente dura, las cadenas aún son frágiles e hidrosolubles; aproximadamente 24 horas después de haber sido mezclado el cemento, predominan las cadenas formadas con iones aluminio, las cuales refuerzan la matriz, haciendo al cemento más resistente, insoluble y de excelentes propiedades físicas; la preponderancia de cadenas formadas con iones calcio $\mathrm{y}$ aluminio marcan las llamadas fases de maduración de este material [27], mientras que en los ionómeros modificados con resinas donde los monómeros de metacrilato y el hidroxietil metacrilato (HEMA) son los responsables de la reacción de polimerización, la reacción ácido-básica, que es lenta, es la responsable de la maduración y resistencia final del cemento [28].

A la hora de manipular el material también se debe tener en cuenta el volumen del líquido y de polvo que se requiere dependiendo de la cavidad por llenar, ya que esta adecuada mezcla nos permitirá una buena 
consistencia del material para así disminuir las burbujas, porosidades y poder aumentar la resistencia a la compresión y durabilidad del material en la pieza dentaria por restaurar. Factores externos, como la temperatura y humedad del ambiente de mezcla, tiempo y técnica de manipulación, también pueden introducir variabilidad inducida por el operador [20].

Con esta investigación científica se pretende identificar el efecto del ácido ortofosfórico al $37 \%$ usado en odontología para el grabado del esmalte y la dentina, sobre la resistencia compresiva de un ionómero de vidrio resino-modificado de fotopolimerización utilizado como base intermedia en operatoria dental, procedimiento que involucra el lavado de dicho ácido con agua.

\section{Materiales y métodos}

Se prepararon cuarenta moldes [21] de polimetilmetacrilato, dentro de los cuales se depositó un ionómero de vidrio resino-modificado que fue mezclado con una espátula metálica y depositado en incrementos no mayores a $1,5 \mathrm{~mm}$, hasta llenarlo completamente (como lo indica la casa fabricante), los cuales tuvieron una medida estándar de $10 \pm 2$ de diámetro y $5 \pm 2$ de espesor según la norma iso (9917-2:2010) [29]. La prueba se realizó en un entorno que mantuvo una temperatura de $(37 \pm 1){ }^{\circ} \mathrm{C}$ y una humedad de al menos el $50 \%$; se utilizaron mezclas frescas para cada muestra.

Se conformaron dos grupos de la siguiente manera: el grupo control (GC) estuvo compuesto por 20 muestras a las que no se les aplicó ácido ortofosfórico al 37\%, y el grupo experimental (GE) estuvo constituido por 20 muestras a las que se les aplico ácido ortofosfórico al $37 \%$ (Proquident ${ }^{\circledR}$ ) durante $15 \mathrm{~s}$ y se lavaron con agua durante $40 \mathrm{~s}$. Diez muestras del grupo control (GC) y diez muestras del grupo experimental (GE) se almacenaron por 48 horas al baño maría, marca Controls, a $37^{\circ} \mathrm{C}$, simulando la temperatura de la cavidad oral, mientras se daba el proceso completo de maduración del ionómero de vidrio. En este proceso, se midió el grosor de las muestras a las 24 horas con un micrómetro, por lo que fueron sacadas del agua y regresadas inmediatamente realizada la medición; las otras 10 muestras del GC y del GE fueron almacenadas durante 15 minutos; después se les midió el grosor con un micrómetro. Las muestras fueron sometidas a una carga de resistencia compresiva, con la utilización de una máquina de prueba universal Instrom, marca Schenck Trebel, modelo OPM serie 12191, y con una capacidad máxima de 120 kilonewton. La velocidad de cruce fue de 0,5 mm/min (según la norma iso 9917-2:2010) [29].

De acuerdo con la resolución $n .^{\circ} 8430$ (4 de octubre de 1993) esta se considera una investigación sin riesgo [30].

Para el análisis estadístico se elaboraron tablas de comparaciones múltiples con la prueba Tukey y ANOvA; el programa estadístico utilizado fue el sPss versión 19 y las tablas se representaron en gráficas de bigotes.

\section{Resultados}

Se evaluaron cuatro grupos de ionómeros de vidrio resino-modificado de fotocurado, cada grupo de diez muestras; todos los grupos fueron sometidos al baño maría, dos grupos con y sin grabado ácido a los $15 \mathrm{~min}$, otros dos grupos con y sin grabado ácido a las 48 horas.

Cuando se comparó la resistencia compresiva en los cuatro grupos, se encontraron diferencias estadísticamente significativas entre los grupos con y sin ácido a los 15 min con respecto a los grupos con y sin ácido a las 48 horas, con un valor de $\mathrm{P}=0,01$ (tabla 1 y figura 1).

Tabla 1. Descripción de la resistencia compresiva en $\mathrm{N}$ y espesor en micras en ionómeros de vidrio resino-modificado de fotocurado con y sin grabado ácido a los 15 minutos y 48 horas

\begin{tabular}{|c|c|c|c|c|c|c|c|c|c|}
\hline \multirow{2}{*}{\multicolumn{2}{|c|}{ Grupos }} & \multirow{2}{*}{$\mathbf{n}$} & \multirow{2}{*}{ Promedio } & \multirow{2}{*}{ DE } & \multicolumn{2}{|c|}{ IC $95 \%$} & \multirow{2}{*}{ Mín. } & \multirow{2}{*}{ Máx. } & \multirow{2}{*}{$\begin{array}{l}\text { Valor P } \\
\text { ANOVA }\end{array}$} \\
\hline & & & & & Lím. inf & Lím. sup & & & \\
\hline \multirow{5}{*}{ 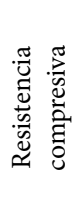 } & 15 min вм sin ácido & 10 & 16,15 & 2,89 & 14,08 & 18,22 & 11,80 & 19,90 & \multirow{4}{*}{0,000} \\
\hline & 15 min вм con ácido & 10 & 16,12 & 2,51 & 14,33 & 17,91 & 12,10 & 20,00 & \\
\hline & 48 horas вм sin ácido & 10 & 7,89 & 5,87 & 3,69 & 12,09 & 0,80 & 16,00 & \\
\hline & 48 horas BM con ácido & 10 & 7,43 & 5,59 & 3,43 & 11,43 & 1,80 & 14,80 & \\
\hline & Total & 40 & 11,90 & 6,08 & 9,95 & 13,84 & 0,80 & 20,00 & \\
\hline
\end{tabular}




\begin{tabular}{|c|c|c|c|c|c|c|c|c|c|}
\hline \multirow{2}{*}{\multicolumn{2}{|c|}{ Grupos }} & \multirow{2}{*}{$\mathbf{n}$} & \multirow{2}{*}{ Promedio } & \multirow{2}{*}{$\mathbf{D E}$} & \multicolumn{2}{|c|}{ IC $95 \%$} & \multirow{2}{*}{ Mín. } & \multirow{2}{*}{ Máx. } & \multirow{2}{*}{$\begin{array}{l}\text { Valor } \mathrm{P} \\
\text { ANOVA }\end{array}$} \\
\hline & & & & & Lím. inf & Lím. sup & & & \\
\hline \multirow{5}{*}{ 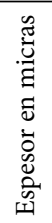 } & 15 min вм sin ácido & 10 & 5,82 & 0,61 & 5,38 & 6,25 & 5,13 & 6,85 & \multirow{4}{*}{0,992} \\
\hline & 15 min вм con ácido & 10 & 5,81 & 0,53 & 5,42 & 6,19 & 5,21 & 6,61 & \\
\hline & 48 horas вм sin ácido & 10 & 5,83 & 0,53 & 5,45 & 6,20 & 5,11 & 6,52 & \\
\hline & 48 horas BM con ácido & 10 & 5,76 & 0,55 & 5,36 & 6,15 & 5,12 & 6,61 & \\
\hline & Total & 40 & 5,80 & 0,53 & 5,63 & 5,97 & 5,11 & 6,85 & \\
\hline
\end{tabular}

Fuente: elaboración propia

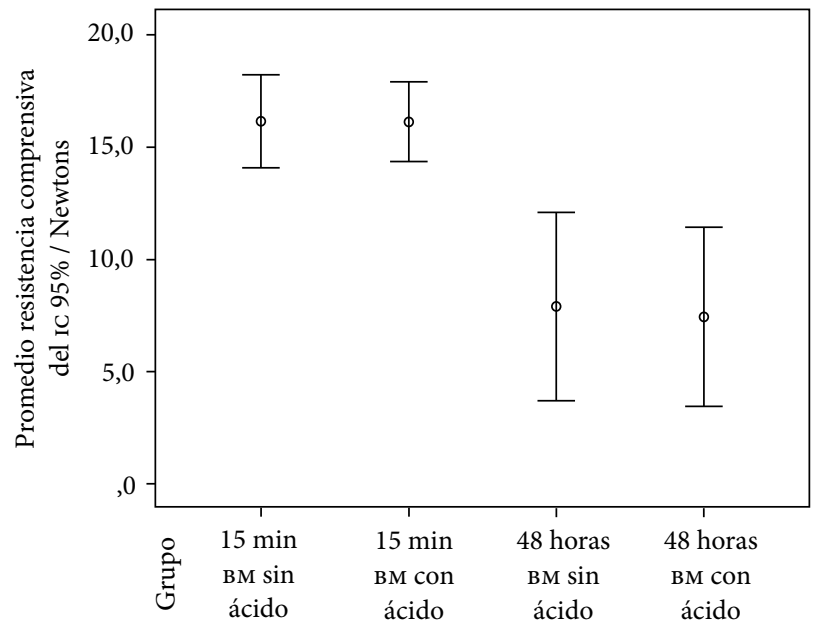

Figura 1. Descripción de la resistencia compresiva en $\mathrm{N}$ del ionómero de vidrio resino-modificado de fotocurado con y sin grabado ácido a las 15 horas y 48 horas

Fuente: elaboración propia

\section{Discusión}

La resistencia a la fractura de los ionómeros de vidrio resino-modificados de foto-polimerización puede verse afectada por diferentes factores tales como: microfiltración, humedad, desecación, mala manipulación del material, poco tiempo de maduración y cargas oclusales.

Los ionómeros de vidrio modificados con resina se caracterizan por tener un mayor tiempo de trabajo, con un sistema rápido, lo que mejora la apariencia y translucidez, así como permite una mayor y más temprana resistencia y fuerza de adhesión al esmalte y a la dentina, presentando una menor micro-filtración comparado con el ionómero convencional. Por otro lado, la rápida reacción de este ionómero es una ventaja para la estabilidad del color, ya que el agua no inhibe la reacción de endurecimiento por la reacción de foto-polimerización. La adhesión de partículas de sílice al polvo de los ionómeros de vidrio resino-modificados ha mostrado mejores resultados después de 24 horas en la resistencia compresiva, en la fuerza tensil-diametral y en la resistencia flexural, así como en la reducción de absorción de agua [31].

Se sabe que la resistencia compresiva (RC) es una propiedad importante en los materiales restaurativos, particularmente en el proceso de masticación [32].

Este estudio reportó que la resistencia compresiva disminuyó en 50\% entre los grupos con y sin ácido a los 15 minutos con respecto a los grupos con y sin ácido a las $48 \mathrm{~h}$, debido al sometimiento del material a la humedad que se puede encontrar en la cavidad oral, resultado que difiere al encontrado en el estudio de Bresciani y colaboradores, en el que se reportó que todos los ionómeros probados en este estudio presentaron un aumento significativo en la resistencia compresiva entre los periodos de 1 hora y 7 días $(\mathrm{p}<0,001)$, pero no se observó diferencia significativa en la resistencia entre los tiempos de 24 horas y 7 días [33], al igual que en el estudio realizado por S. B. Mitra, en el cual se registró que no hubo diferencias estadísticamente significativas en la resistencia compresiva del ionómero inmediatamente después de ser fotocurado y después de ser almacenado a $37^{\circ} \mathrm{C}$ [34].

Estudios anteriores no aceptaban el grabado ácido sobre el ionómero de vidrio por el posible deterioro de la superficie del cemento, además de posibles fallas en la cohesión. Posteriormente, McLean y colaboradores propusieron grabar el ionómero por 60 segundos para lograr una traba mecánica entre las resinas y el cemento. Pamir y colaboradores (2012) reportaron que no se observó al microscopio electrónico deterioro subsuperficial de las superficies de cemento de ionómero de vidrio donde se aplicó el grabado ácido. Además, el proceso de grabado ácido mejoró la fuerza de adhesión de la resina compuesta, siendola concentración más óptima el ácido fosfórico al 35\% durante 30 segundos. No hubo diferencias estadísticamente significativas en la fuerza adhesiva entre los autograbadores y los adhesivos con grabado y lavado. La fuerza adhesiva de la resina al ionómero convencional fue significativamente más baja con respecto al ionómero resino-modificado (se observaron al microscopio superficies fracturadas en la interfase entre el 
adhesivo y el ionómero convencional), en contraste con una muy buena adhesión observada entre el ionómero resino-modificado y el sistema adhesivo. Se ha sugerido que la similitud en la composición de ambos materiales y el iniciador de radicales libres del proceso de polimerización podrían ser responsables de una mayor fuerza adhesiva [35, 36].

Otro aspecto importante por considerar del ionómero de vidrio resino-modificado de foto-polimerización es la filtración marginal - ya que esta dependerá del buen sellado marginal de las cavidades, la conservación de la dentina, la pulpa y otros tantos componentes de las piezas dentarias-, además del éxito en cuanto a la duración y estado del material de obturación elegido para la restauración final.

Con los hallazgos encontrados en este estudio se puede determinar que el grabado ácido se puede realizar sobre el ionómero de vidrio, debido a que el ácido ortofosfórico y su lavado no generan alteración en la resistencia compresiva de este.

\section{Conclusiones}

- La aplicación del ácido fosfórico durante 15 segundos y el lavado durante 40 segundos no afectan la resistencia compresiva del ionómero de vidrio resino-modificado.

- Hubo una diferencia estadísticamente significativa entre los dos grupos (15 minutos y 48 horas), pues se observó disminución de la resistencia compresiva aproximadamente del $50 \%$ porque las muestras estuvieron almacenadas al baño maría, al simular la temperatura de la cavidad oral, debido a la sensibilidad del cemento a la humedad. No se debe dejar el material expuesto a la cavidad oral puesto que se afectará la resistencia compresiva del material y, por ende, su estabilidad y duración en boca, lo que podría conducir a un posible fracaso en el tratamiento realizado.

Cuando se comparó la homogeneidad de las 40 muestras usadas en el estudio, divididas en cuatro grupos, no se encontraron diferencias en su espesor (figura 2).

\section{Referencias}

[1] Anusavice KJ. Ciencia de los materiales dentales. 11 a ed. España: Elsevier; 2008.

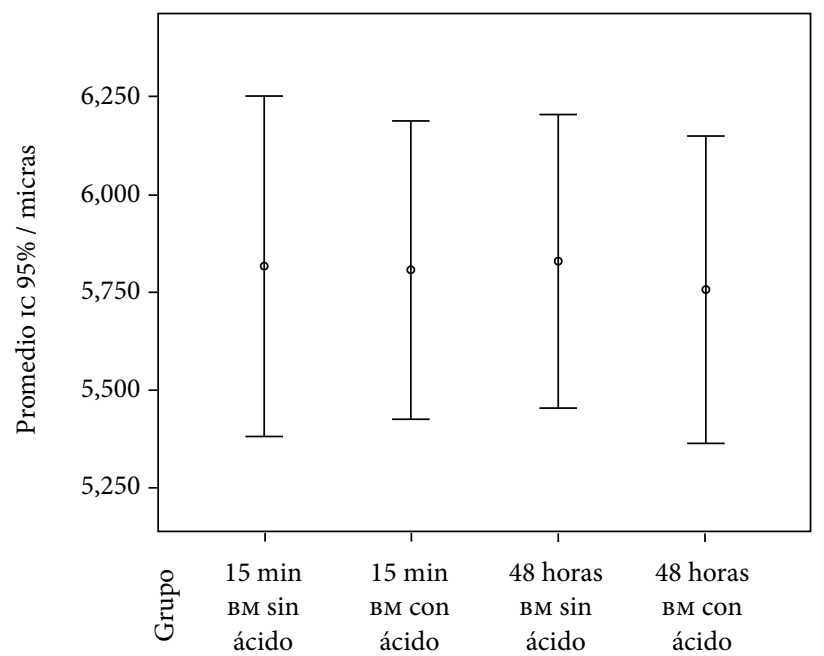

Figura 2. Homogeneidad de las muestras

Fuente: elaboración propia

[2] Es-Sounia M, Fischer-Brandiesa H, Zaporojshenkoc V, Es-Sounib M. On the interaction of polyacrylic acid as a conditioning agent with bovine enamel. Biomaterials. 2002; 23: 2871-8.

[3] Nezu T, Winnik F. Interaction of water-soluble collagen with poly (acrylic acid), Biomaterials. 2000; 21: 415-9.

[4] Gladys S, Van Meerbeek B, Braem M, Lambrechts P, Vanherle G. Comparative physico-mechanical characterization of new hybrid restorative materials with conventional glass-ionomer and resin composite restorative materials. J Dent Res. 1997; 76: 883.

[5] Bhalla M, Patel D, Shashikiran ND, Mallikarjuna RM, Nalawade TM, Reddy HK. Effect of light-emitting diode and halogen light curing on the micro-hardness of dental composite and resin-modified glass ionomer cement: An in vitro study. J Indian Soc Pedod Prev Dent [Internet] 2012 [Consultado: abril $18 \mathrm{del}$ 2013]. Disponible en: http://www.jisppd.com/text.asp?2012/30/3/201/105011

[6] Roberson TM, Heymann HO, Swift EJ. Arte y ciencia de la odontología conservadora. $5^{a}$. ed. España: Elsevier; 2007.

[7] Abate PF, Borgia E, Busato A, Carvalho R, Corts JP, Costa CA et al. Adhesión en odontología restauradora. 2a. ed. Madrid: Editorial Médica; 2010.

[8] Barrancos J. Operatoria dental. Integración Clínica. $4^{\text {a }}$. ed. Buenos Aires: Editorial Médica Panamericana; 2008.

[9] Hidalgo R, Méndez M. Técnica Sándwich con acondicionamiento ácido selectivo empleando ionómeros. Acta Odontol Venez. 2009; 39(3): 1-24.

[10] Berástegui J, Dolset M, Solans R. Estudio piloto del pulido de ionómeros de vidrio mediante microscopio 
electrónico de barrido. Dental World. [Internet]. [Consultado: 12 de abril del 2010]. Disponible en: http://gbsystems.com/papers/general/art5.htm

[11] Ahluwalia P, Chopra S, Thomas AM. Strength characteristics and marginal sealing ability of chlorhexidinemodified glass ionomer cement: an in vitro study. J Indian Soc Pedod Prev Dent. 2012; 30: 41-6.

[12] Ochoa CA, Rueda K, Pulido E. Utilización del ionómero de vidrio como material de obturación coronal temporal. [Internet] 2006 [Consultado: 30 de septiembre del 2010]. Disponible en: http://www.javeriana.edu.co/ academiapgendodoncia/i_a_revision07.html

[13] Geurtsen W. Substances released from dental resin composites and glass onomer cements. Eur J Oral Sci. 1998; 106(2): 687-95.

[14] Kurata S, Umemoto K. Characteristics of acrylic acidmodified temperature-responsive polymers and application as cement liquid. Dent Mater J. 2011; 30(1): 115-20.

[15] Moscardó A, Romero B, Ferrandis L, Camps I. Influencia de la técnica de grabado total en el sellado marginal en cavidades clase V obturadas con compómeros. Electronic Journal of Dental Reserch [artículo en línea]. 1997. [Consultado: 24 de abril del 2010]. Disponible en: http://www.uv.es/pascuala/ejdr/Art00006.htm

[16] Fleming G, Farooq A, Barralet J. Influence of powder/ liquid mixing ratio on the performance of a restorative glass-ionomer dental cement. Biomaterials. 2003; 24: 4173-9.

[17] Serpil K, Nilgün A, Hatice Nur Ö, Hayati-Murat A. Effectiveness of surface protection for glass-ionomer, resin-modified glass-ionomer and polyacid-modified composite resins. Dent Mater J. 2009; 28(1): 96-101.

[18] Berzins DW, Abey S, Costache MC, Wilkie CA, Roberts HW. Resin-modified Glass-ionomer Setting Reaction Competition. J Dent Res. 2010; 89(1): 82-6.

[19] Smith DC. Dental cements. Adv Dent Res. 1988; 2(1): 134-41.

[20] Kleverlaan C, van Duinen R, Feilzer A. Mechanical properties of glass ionomer cements affected by curing methods. Mater Dent. 2004; 20(1): 45-50.

[21] Mallmann A, Oliveira Ataíde JC, Amoedo R, Rocha PV, Borges Jacques L. Compressive strength of glass ionomer cements using different specimen dimensions. Braz Oral Res. 2007; 21(3): 204-8.

[22] De Guzmán AR. Evaluación clínica de un ionómero de vidrio modificado en odontopediatría. Acta Odontol Venez. [Internet] 2001. [Consultado: 2 de agosto del 2010].Disponibleen:http://www.actaodontologica.com/ ediciones/2001/3/evaluacion_ionomero_vidrio_modificado.asp
[23] Della A, Pinzetta C, Rosa V. Effect of acid etching of glass ionomer cement surface on the microleakage of sandwich restorations. J Appl Oral Sci. 2007; 15(3): 230-4.

[24] Anstice H, Nicholson J, McCabe J. The Effect of Using Layered Specimens for Determination of the Compressive Strength of Glass-ionomer Cements. J Dent Res. 1992; 71(12): 1871-4.

[25] Rekha CV, Varma B, Jayanthi. Comparative evaluation of tensile bond strength and microleakage of conventional glass ionomer cement, resin modified glass ionomer cement and compomer: an in vitro study. Contemp Clin Dent. 2012 Jul; 3(3): 282-7.

[26] Doméjean S, Banerjee A, Gaucher C, Milètic I,Basso M, Reich E et al. Plan de tratamiento de mínima intervención. J Minim Interv Dent. 2009; 2(2): 275-97.

[27] García H, Cuartas M, Castaño A, Llano E. Revisión sistemática del efecto del ácido fosfórico usado en resinas compuestas sobre la desmineralización dentinal. Rev Fac Odontol Univ Antioq. 2004; 16(1/2): 60-9.

[28] Nilufer C, Beriata, Dilek Nalbantb, Water Absorption and HEMA release of resin-modified glass-ionomers. Eur J Dent. 2009; 3(4): 267-72.

[29] International Organization for Standardization. ISO 9917-2. Dentistry-water-based cements. Part 2: Resinmodified cements. Switzerland: ISO; 2010.

[30] Colombia. Ministerio de Salud. Resolución número 8430 de 1993 por la cual se establecen las normas científicas, técnicas y administrativas para la investigación en salud. Bogotá: El Ministerio; 1993.

[31] Lihua E, Irie M, Nagaoka N, Yamashiro T, Suzuki K. Mechanical properties of a resin-modified glass ionomer cement for luting: effect of adding spherical silica filler. Dental Mater J. 2010; 29(3): 253-61.

[32] Shen C, Grimaudo N. Effect of hydration on the biaxial flexural strength of a glass ionomer cement. Dent Mater. 1994; 10(3): 190-5.

[33] Bresciani E, Barata T, Facundes TC, Adachi A, Terrin MM, Navarron MF. Resistencia compresiva y tensil diametral de los cementos ionómero vítreos. J Minim Interv Dent. [Internet] 2008 [Consultado: 9 de octubre del 2011]. Disponible en: http://www.miseeq. com/s-1-2-4.pdf

[34] Mitra SB. Adhesion to dentin and physical properties of a light-cured glass- ionomer liner/Base. J Dent Res. 1991; 70(1): 72-4.

[35] Pamir T, Şen BH, Evcin Ö. Effects of etching and adhesive applications on the bond strength between composite resin and glass-ionomer cements, J Appl Oral Sci. 2012; 20(6): 636-42.

[36] Nujella BPS, Choudary MT, Reddy SP, Kumar MK, Gopal T. Comparison of shear bond strength of aesthetic restorative materials. Contemp Clin Dent. 2012; 3: 22-6. 